\title{
ON THE VOLUME ELEMENTS ON A MANIFOLD ${ }^{1}$ )
}

\author{
BY \\ JƯRGEN MOSER
}

1. We consider a closed connected $n$-dimensional manifold $M$. By a volume element we mean a differential form of degree $n$ of odd kind, everywhere positive $\left({ }^{2}\right)$. In local coordinates $x_{1}, \cdots, x_{n}$ such a volume element $\tau$ takes the form

$$
\tau=g(x) d x \text { where } d x=d x_{1} \wedge d x_{2} \wedge \cdots \wedge d x_{n}
$$

and $g(x)>0$.

Clearly any two such volume elements $\tau$, are related by

$$
\sigma=f \tau
$$

where $f$ is a positive scalar function on $M$. Throughout we assume $M$ to be a $C^{\infty}$ manifold and accordingly assume $g, f$ to be $C^{\infty}$ functions.

The problem to be studied is that of the equivalence of such volume elements on a manifold $M$ under $C^{\infty}$-automorphisms of $M$. If $\phi$ is such an orientation preserving automorphism of $M$ onto itself a volume element $\tau$ is transformed into another volume element $\phi^{*} \tau$ such that

$$
\int_{\phi(S)} \phi^{*} \tau=\int_{S} \tau \quad \text { for } S \subset M .
$$

Any two volume elements $\tau, \sigma$ which can be transformed into each other in this manner will be called equivalent: $\tau \sim \sigma$. Clearly, if $\tau \sim \sigma$ then their total volume

$$
\int_{M} \tau=\int_{M} \sigma
$$

is invariant under such an automorphism. The statement to be proven asserts that their total volume is the only invariant.

THEOREM. If $\tau, \sigma$ are any two volume elements on a compact, connected manifold then

Received by the editors December 21, 1964.

(1) This work has been supported by the contract NONR-285 (46).

(2) Originally our statement referred to $n$-forms on orientable manifolds only. Professor Calabi kindly pointed out that the result could be extended to nonorientable manifolds if one uses the concept of "odd" forms which was introduced by de Rham, Variétés différentiables, $\S 5$. Such $n$-forms of odd kind are characterized by the property that they multiply by $|J|$ under coordinate transformation when $J$ denotes the Jacobian of the transformation. 
where $\lambda$ is the constant

$$
\sigma \sim \lambda \tau
$$

$$
\lambda=\int_{M} \sigma / \int_{M} \tau .
$$

Here it is understood that the automorphism $\phi$ of $M$ which takes $\sigma$ into $\lambda \tau$ is in $C^{\infty}$.

The above statement could be formulated somewhat more generally: Consider two manifolds $M, N$ with volume elements $\tau, \sigma$ and assume that $M, N$ can be mapped onto each other by a differentiable homeomorphism $\phi: M \rightarrow N$. If

$$
\int_{M} \tau=\int_{N} \sigma
$$

then there exists a differentiable homeomorphism of $M$ onto $N$ taking also $\tau$ into $\sigma$. This problem was raised by R. Palais in a conversation which gave rise to this note.

The proof which follows is quite elementary. It provides the construction of an orientation preserving automorphism homotopic to the identity.

This will be done by reducing the theorem to a local statement where $f$ differs from 1 in one coordinate patch only. The construction of a diffeomorphism in this case can be given by simple integration.

2. To reduce the problem we observe that it suffices to prove the theorem for any two volume elements $\tau, \sigma=f \tau$ for which

$$
|f-1|<\varepsilon
$$

where $\varepsilon$ is any preassigned positive number. Namely, setting

$$
\sigma_{t}=f^{t} \tau \text { for } 0 \leqq t \leqq 1
$$

it would follow that

$$
\sigma_{t^{\prime \prime}} \sim \text { const } \sigma_{t^{\prime}}
$$

whenever $\left|t^{\prime \prime}-t^{\prime}\right|<\delta$ is sufficiently small, e.g. $\delta=\log (1+\varepsilon) / \max |\log f|$. Hence in finitely many steps one obtains that $\sigma=\sigma_{1} \sim c \sigma_{0}=c \tau$ where $c$ is some positive constant. Of course, we can always assume that this constant is normalized to one, i.e. we shall from now on assume

$$
\int_{M} \sigma=\int_{M} \tau=1
$$

By a similar continuation argument we shall reduce the theorem to a local one. For this purpose let the manifold $M$ be covered by open sets $U_{0}, U_{1}, \cdots, U_{m}$ which can be mapped one to one onto the unit cube 


$$
Q: 0<x_{v}<1 \quad(v=1, \cdots, n)
$$

of the Euclidean space.

LEMMA 1. If $g$ is a continuous function on $M$ which satisfies

$$
\int_{M} g \tau=0 .
$$

Then there exists a decomposition of $g$ into

$$
g=\sum_{j=0}^{m} g_{j}
$$

where $g_{j}$ has support in $U_{j}$ and satisfies

$$
\int g_{j} \tau=0
$$

Moreover, $\left|g_{j}\right| \leqq c$ sup $|g|$ where $c$ depends on $M$ and the covering only. If $g \in C^{r}$ so is $g_{j} \in C^{r}$.

This lemma allows us to reduce the proof of the theorem to a function $f$ which differs from 1 in one patch $U_{j}$ only. Namely, applying Lemma 1 to $g=f-1$, we interpolate 1 and $f$ by

$$
f(t ; p)=1+\sum_{j} t_{j} g_{j} ; \quad p \in M
$$

where $t=\left(t_{0}, t_{1}, \cdots, t_{m}\right)$ ranges over $0 \leqq t_{j} \leqq 1$. For $t=(0, \cdots, 0)$ one has $f(t, p)=1$ and for $t=(1, \cdots, 1)$ the function $f(t, p)$ agrees with the given function $f$. For all values of $t$ the total volume is fixed and equal to one. Finally, we choose in (2) $\varepsilon<(m+1)^{-1} c^{-1}$ so that

$$
f>1-(m+1) c \varepsilon>0 .
$$

We can connect the two corners $t=(0, \cdots, 0)$ and $t=(1, \cdots, 1)$ of the cube $0 \leqq t_{j} \leqq 1$ by going along $(m+1)$ edges. If $t^{\prime}, t^{\prime \prime}$ represent the endpoints of such an edge one sees that

$$
f\left(t^{\prime \prime}, p\right)-f\left(t^{\prime}, p\right)
$$

has support in one patch, say $U_{j}$. Hence if

$$
\sigma_{t}=f(t, p) \tau
$$

one sees that

$$
\sigma_{t^{\prime \prime}}=h \sigma_{t^{\prime}}
$$


where

$$
h=\frac{f\left(t^{\prime \prime}, p\right)}{f\left(t^{\prime}, p\right)}
$$

is different from 1 in $U_{j}$ only. If the equivalence of such volume elements is shown it follows that $\sigma_{t^{\prime \prime}} \sim \sigma_{t^{\prime}}$ and successively $\sigma \sim \tau$. Therefore it suffices to show the equivalence of $\tau$ and $\sigma=f \tau$ in case $f-1$ has support in one coordinate patch only. This can be expressed in coordinates as follows:

LEMMA 2. Let

$$
\tau=g(x) d x, \quad \sigma=h(x) d x,
$$

be two volume elements in the unit cube $Q \subset R^{n}$ in $Q$, where $g, h$ are positive functions for which $g-h$ has support inside $Q$. If

$$
\int_{Q} g d x=\int_{Q} h d x
$$

then there exists a coordinate transformation

$$
y=u(x)
$$

which maps the unit cube one to one onto itself such that

$$
g(u(x)) \operatorname{det} u_{x}=h(x)
$$

or

$$
g(y) d y=h(x) d x
$$

and such that $u(x)=x$ near the boundary of $Q$.

Thus the theorem is reduced to the proof of the two lemmata, which we present now.

3. To prove Lemma 1 we shall make use of a partition of unity $\phi_{j} \geqq 0$, where each function $\phi_{j}$ has support in $U_{j}$. Clearly $g \phi_{j}$ will also have support in $U_{j}$ but the condition (4) requires special attention. Since $M$ is connected, the $U_{j}$ can always be ordered in such a manner that for every $k=1, \cdots, m$ the patch $U_{k}$ intersects $\bigcup_{j<k} U_{j}$. We pick an integer $\rho(k)<k$ such that

$$
U_{k} \cap U_{\rho(k)}
$$

is not empty. We introduce the matrix $\alpha=\left(\alpha_{j k}\right)$ by

$$
\alpha_{j k}=\left\{\begin{aligned}
1 & \text { for } j=k \\
-1 & \text { for } j=\rho(k) \\
0 & \text { otherwise for } 0 \leqq j \leqq m, 1 \leqq k \leqq m
\end{aligned}\right.
$$


Each of the $m$ columns contains exactly one pair $+1,-1$ so that

$$
\sum_{j=0}^{m} \alpha_{j k}=0
$$

To construct the functions $g_{j}$ we need functions $\eta_{k}(k=1, \cdots, m)$ with support in the open set described by (6) and such that

$$
\int \eta_{k} \tau=1
$$

Such functions can always be found. Then the $g_{j}$ of Lemma 1 can be represented in the form

$$
g_{j}=g \phi_{j}-\sum_{k=1}^{m} \lambda_{k} \alpha_{j k} \eta_{k}
$$

We verify that the $g_{j}$ satisfy all requirements if the $\lambda_{1}, \cdots, \lambda_{m}$ are chosen appropriately. On account of (7) the $g_{j}$ add up to $g$. Moreover, for a given $j$ we have $\alpha_{j k} \neq 0$ only if $k=j$ or $\rho(k)=j$ and for those $k$ the support of $\eta_{k}$ lies in

$$
U_{k} \cap U_{\rho(k)} \subset U_{j}
$$

which shows that also $g_{j}$ has support in $U_{j}$. Finally, to satisfy (4) we determine the $\lambda_{1}, \cdots, \lambda_{m}$ from the resulting $m+1$ equations

$$
\sum_{k=1}^{m} \lambda_{k} \alpha_{j k}=\int_{M} g \phi_{j} \tau .
$$

On account of (7) and

$$
\int_{M} g \tau=\sum_{j=0}^{m} \int_{M} g \phi_{j} \tau=0
$$

the first equation $(j=0)$ is redundant. The other $m$ equations are uniquely solvable since the rank of $\alpha$ is $m$. The additional statement of the lemma is immediate from this construction and the proof of Lemma 1 is complete.

The above construction has an interpretation in graph theory: One may interpret $U_{j}$ as nodes of a graph connecting $U_{j}$ and $U_{k}$ by a branch $U_{j} \cap U_{k}=D_{j k}$ if this set is nonempty. The graph so obtained is connected since $M$ is. The above matrix $\alpha_{j k}$ corresponds to the incidence matrix of a maximal tree in this graph. It is well known that such a tree has $m$ branches if the graph has $m+1$ nodes.

Finally we prove Lemma 2 and solve the differential equation (5). To avoid the nonlinear character of the equation we represent $u$ in the form $w^{-1} \circ v$ with

$$
z=v(x), \quad z=w(y),
$$

where $v, w$ satisfy 


$$
\operatorname{det} v_{x}=\operatorname{ch}(x) ; \operatorname{det} w_{y}=c g(y) \text {. }
$$

Clearly this implies that $u=w^{-1} \circ v$ satisfies (5). In order that $u(x)=x$ near $\partial Q$ we shall arrange it so that $v(x)=w(x)$ near the boundary of $Q$.

We shall give the construction for $h$ and find $v(x)$ in the special form

$$
z_{v}=v_{v}\left(x_{1}, \cdots, x_{v}\right), \quad v=1, \cdots, n,
$$

and so that the faces of the cube are preserved, i.e. that

$$
v_{v}\left(x_{1}, \cdots, x_{v-1}, \varepsilon\right)=\varepsilon \quad \text { for } \varepsilon=0,1 .
$$

Without loss of generality we can assume that $c=1$ and then we see from (8) that the requirement for $v_{v}$ is

$$
\prod_{v=1}^{n} \frac{\partial v_{v}}{\partial x_{v}}=h
$$

For this purpose we factor $h=h\left(x_{1}, \cdots, x_{n}\right)$ into

such that

$$
\operatorname{ch}(x)=\prod_{v=1}^{n} h_{v}\left(x_{1}, \cdots, x_{v}\right)
$$

$$
\int_{0}^{1} h_{v}\left(x_{1}, \cdots, x_{v-1}, t\right) d t=1 .
$$

This is possible and in a unique way as one sees by induction with respect to $n$. One has

$$
h_{n}(x)=h(x) / \int_{0}^{1} h\left(x_{1}, \cdots, x_{n-1}, t\right) d t .
$$

It suffices to find a transformation $v$ for one of the above factors, say $h_{\rho}$. This requires the solution of

$$
\operatorname{det} v_{x}=h_{\rho}\left(x_{1}, \cdots, x_{\rho}\right)
$$

which is given explicitely by

$$
v_{v}=x_{v} \text { for } v \neq \rho ; \quad v_{\rho}=\int_{0}^{x_{\rho}} h_{\rho}\left(x_{1}, \cdots, x_{\rho-1}, t\right) d t .
$$

Clearly, this mapping takes $\bar{Q}$ onto itself. One verifies from the construction: if $g-h$ has its support in $D \subset Q$ then the corresponding solutions $v, w$ agree outside any cube containing $D$. Hence $u=w^{-1} \circ v$ has all desired properties. Incidentally, if $h$ is in $C^{r}$ so is $v$ in $C^{r}$ but not necessarily in $C^{r+1}$. This proves Lemma 2 and thus the above theorem.

4. We sketch an alternate proof of the above theorem which also leads to an 
analogous statement for closed 2-forms. In this proof we shall require Hodge's decomposition theorem for forms on Riemannian manifolds: Every closed $p$-form $\omega$ can be represented in the form

$$
\omega=d \alpha+h
$$

where $h$ is a harmonic $p$-form and $\alpha$ some $(p-1)$-form. In order to make the choice of $\alpha$ unique we shall require that $\alpha=\delta \beta$ with some $p$-form $\beta$. Moreover, the harmonic form $h$ vanishes if and only if all periods of $\omega$ are zero.

The above normalization of $\alpha$ requires a metric on the manifold which we introduce in an arbitrary manner. The concept of a harmonic form and the differential operator $\delta$ depends on the choice of this metric $\left({ }^{3}\right)$.

We formulate the following statement at once for $n$-forms and for 2-forms of odd kind. We call a 2-form nondegenerate if $\omega \wedge \omega \wedge \cdots \wedge \omega=\omega^{m}=0$ where the dimension of the manifold $n=2 m$ is even.

THEOREM 2. Let $\tau_{t}$ be a family of closed 2-or $n$-forms of odd kind which are nondegenerate for $0 \leqq t \leqq 1$ and with fixed periods, i.e.

$$
\int_{c} \tau_{t}=\int_{c} \tau_{0}
$$

for every 2-or $n$-dimensional cycle c on $M$. Then there exists an automorphism $\phi_{t}$ such that

$$
\phi_{t}^{*} \tau_{t}=\tau_{0}
$$

and $\phi_{0}$ is the identity mapping.

Proof. Instead of determining the mapping $\phi_{t}$ directly we first shall find a vectorfield $u_{t}$ on $M$ from which we shall recover by solving the ordinary differential equation $\left({ }^{4}\right)$

$$
\frac{d}{d t} \phi_{t}=u_{t} \circ \phi_{t}
$$

This has the advantage that $u_{t}$ is obtained from linear equations while $\phi_{t}$ satisfies some nonlinear equations.

To derive the condition for $u_{t}$ we differentiate the equation (10) with respect to $t$ and use the following formula:

$$
\frac{d}{d t}\left(\phi_{t}^{*} \omega\right)=\phi_{t}^{*}\left\{(d \omega) \downarrow u_{t}+d\left(\omega \downarrow u_{t}\right)\right\}
$$

(3) For the definition of $\delta$ and the above decomposition theorem we refer again to de Rham's book, in particular, Theorem 25, p. 168.

(4) In the language of fluid dynamics this representation corresponds to the use of Euler coordinates instead of Lagrange coordinates. 
where $\omega\lrcorner u$ denotes the inner product of a differential $\omega$ and a vectorfield $u\left({ }^{5}\right)$. Hence if we denote $(d / d t) \tau_{t}$ by $\dot{\tau}_{t}$ we find from (10)

$$
0=\frac{d}{d t}\left(\phi_{t}^{*} \tau_{t}\right)=\phi_{t}^{*}\left\{\dot{\tau}_{t}+d\left(\tau_{t} \sqsupset u_{t}\right)\right\}
$$

since $d \tau_{t}=0$ by assumption.

Applying the decomposition (9) to $\omega=\tau_{t}$ we have

$$
\dot{\tau}_{t}=d \alpha_{t}+h_{t}
$$

Since we assumed that the periods of $\tau_{t}$ were independent of $t$, those of $\dot{\tau}_{t}$ are zero and hence $h_{t}=0$. Thus

$$
\dot{\tau}_{t}=d \alpha_{t}
$$

and the form $\alpha_{t}$ is uniquely determined by the normalisation described above. The vectorfield $u_{t}$ will now be determined from the linear equation

$$
\left.\tau_{t}\right\lrcorner u_{t}=-\alpha_{t}
$$

which defines $u_{t}$ uniquely since $\tau_{t}$ was assumed to be nondegenerate. To show this we write the inner product in coordinate representation:

For

$$
\tau_{t}=\sum_{k, l=1}^{n} a_{k l}(x) d x_{k} d x_{l} \text { or } a(x) d x_{1} \cdots d x_{n}
$$

with $a_{k l}+a_{l k}=0$ and a vectorfield

$$
u_{t}=\sum_{k=1}^{n} u_{k} \frac{\partial}{\partial x_{k}}
$$

one has

$$
\left.\tau_{t}\right\rfloor u_{t}=\sum_{k, l=1}^{n} a_{k l} u_{k} d x_{l} \text { or } a(x) \sum_{k=1}^{n}(-1)^{k-1} u_{k} d x_{1} \cdots d \hat{x}_{k} \cdots d x_{n} .
$$

In both cases the nondegeneracy ensures that the coefficients of $u_{t}$ are uniquely determined by the coefficients of $\alpha_{t}$. At this point it is crucial that the form $\alpha_{t}$ has $n$ components which occurs for 1 - and $(n-1)$-forms only. For this reason the above theorem holds only for 2- and $n$-forms $\tau_{t}$.

Having determined $u_{t}$ we solve the differential equation (11)-which presents no difficulty on a compact manifold. The solution $\phi_{t}$ satisfies equation (12) which after integration leads to the statement (10) of the theorem.

(5) See for example, S. Sternberg, Lectures on differential geometry, Prentice-Hall, 1964, p. 22; the above formula can be found on p. 102 (formula (1.9)) of this book. 
The statement concerning 2-forms was also suggested by R. Palais. Unfortunately it seems very difficult to decide when two 2-forms which are closed, belong to the same cohomology class and are nondegenerate can be deformed homotopically into each other within the class of these differential forms. If the last condition of nondegeneracy were dropped this is no problem since every 2-form can be connected to the unique harmonic form which lies in the same cohomology class.

Namely, if

$$
\tau=d \alpha+h
$$

the deformation is provided by

$$
\tau_{t}=t d \alpha+h \quad(0 \leqq t \leqq 1) .
$$

Then $d \tau_{t}=0, \tau_{t}$ belongs to a fixed cohomology class, but the nondegeneracy condition cannot be ensured.

Even for the simple example of the $n$-dimensional complex projective space $P^{n}$ this problem could not be decided by us. If one represents the space by $n+1$ complex coordinates $z_{0}, z_{1}, \cdots, z_{n}$ satisfying

$$
\sum_{k=0}^{n}\left|z_{k}\right|^{2}=1
$$

and identifying all vectors $e^{i \alpha}\left(z_{0}, \cdots, z_{n}\right)$ for different real $\alpha$ one has the nondegenerate real 2-form

$$
\tau=\frac{1}{2 i} \sum_{k=0}^{n} d z_{k} \overline{d z}_{k}
$$

on $P^{n}$ (considered as $2 n$-dimensional real manifold). But for $n>2$ it has not been decided whether every closed and nondegenerate $2-$ form on $P^{n}$ is equivalent to $\lambda \tau$. This seems to be a difficult topological problem.

Finally we mention that this second approach leads to a particularly simple proof of Darboux's theorem( $\left.{ }^{6}\right)$ about a local normal form for nondegenerate closed 2 forms.

NEW YORK UNIVERSITY, NEW YoRK, New YoRK

(6) See, for example, S. Sternberg's book, quoted above, on p. 140, Theorem 6.1. 